\title{
ASYMPTOTICS OF ENDS OF CONSTANT MEAN CURVATURE SURFACES WITH BUBBLETONS
}

\author{
SHIMPEI KOBAYASHI
}

(Communicated by Richard A. Wentworth)

\begin{abstract}
A constant mean curvature surface with bubbletons is defined by the loop group action on the set of extended framings for constant mean curvature surfaces by simple factors. Classically such surfaces were obtained by the transformation of tangential line congruences, the so-called BianchiBäcklund transformations.

In this paper, we consider constant mean curvature surfaces with Delaunay ends in three-dimensional space forms $\mathbb{R}^{3}, S^{3}$ and $H^{3}$ and their surfaces with bubbletons for which the topology is preserved. We show that the ends of such surfaces are again asymptotic to Delaunay surfaces.
\end{abstract}

\section{INTRODUCTION}

It is well known that the bubbleton surface is a Bianchi-Bäcklund transformation, which is a transformation between surfaces using line congruences [1], of a round cylinder in $\mathbb{R}^{3}[8]$.

C.-L. Terng and K. Uhlenbeck [9] showed that such geometric transformations can be reformulated as the action of loop groups on the set of the extended framings for surfaces by particular elements, which are called "simple factors".

Recently, using dressing action by these simple factors and the generalized Weierstrass type representation for constant mean curvature (CMC) surfaces developed by J. Dorfmeister, F. Pedit and H. Wu (the DPW method [2]), M. Kilian, N. Schmitt and I. Sterling [5] discussed a dressing action by simple factors for CMC $n$-noids (CMC-immersions from an $n$-punctured sphere $(n \geq 3))$ in $\mathbb{R}^{3}$. In general, such a dressing action is only defined on the universal cover of a surface, and thus does not preserve the topology of the original surface. They proved the existence of particular simple factors for which the dressing action does indeed preserve the topology of the CMC $n$-noids. The existence of such particular simple factors was already known for round cylinders and Delaunay surfaces [6], [8].

In this paper, we define the "CMC surface with bubbletons" as the surface produced by a dressing action by simple factors of that CMC surface (see Definition 3.1). We use this slightly cumbersome expression because it is more general than classical bubbletons, which were derived only from round cylinders. For example, we have Delaunay surfaces with bubbletons in space forms as in [6] (including

Received by the editors April 11, 2006 and, in revised form, February 15, 2007.

2000 Mathematics Subject Classification. Primary 53A10.

(c) 2007 American Mathematical Society Reverts to public domain 28 years from publication 
classical bubbletons), and $n$-noids with bubbletons in $\mathbb{R}^{3}$ as in [5]. By numerical experimentation in [6] and [5], properties of the ends of CMC surfaces with bubbletons seemed to be asymptotically the same as properties of the ends of the original surfaces. (See Figures 1 and 2.)

In the present paper, we consider CMC surfaces with Delaunay ends, and consider their surfaces with bubbletons for which the topology is preserved in threedimensional space forms. We prove that ends of these CMC surfaces with bubbletons again converge to Delaunay surfaces (Theorem 3.1). As a corollary, we show that Delaunay surfaces with bubbletons and $n$-noids $(n \geq 3)$ with bubbletons for which the topology is preserved have Delaunay ends.

The paper is organized as follows: In Section 2, we give basic notations and results for CMC surfaces in space forms. In Section 3, we give the main result Theorem 3.1 of this paper, the convergence of the ends of CMC surfaces with bubbletons. In Section 4 , we apply Theorem 3.1 to Delaunay surfaces with bubbletons and $n$-noids with bubbletons.
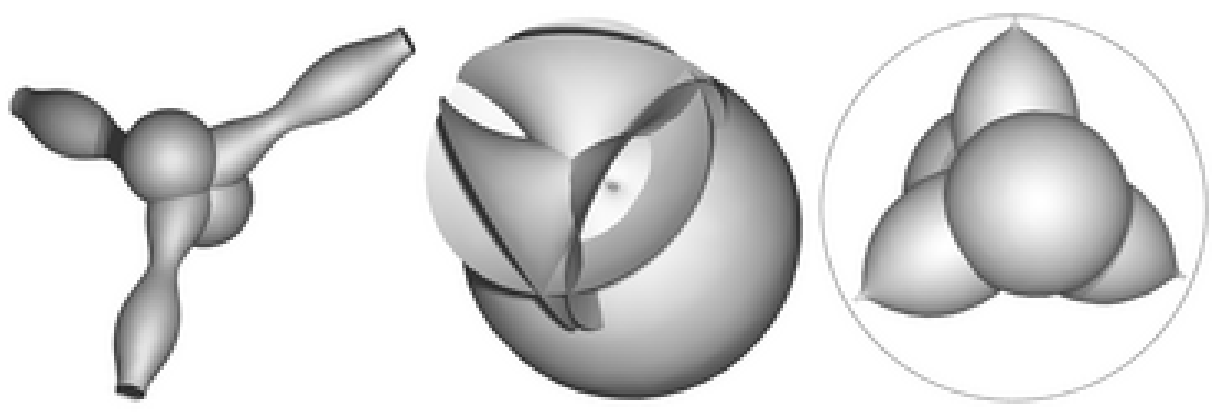

Figure 1. Trinoids with bubbletons in $\mathbb{R}^{3}, S^{3}$ and $H^{3}$. Images are created by Nick Schmitt using CMCLab [7].

\section{Preliminaries}

2.1. Loop groups. We first introduce loop groups and loop algebras. Let $C_{r}:=$ $\{\lambda \in \mathbb{C}|| \lambda \mid=r\}$ be the circle of radius $r$ with $r \in(0,1]$, and let $D_{r}:=\{\lambda \in$ $\mathbb{C}|\quad| \lambda \mid<r\}$ be the open disk of radius $r$. We denote the closure of $D_{r}$ by $\overline{D_{r}}:=\{\lambda \in \mathbb{C}|| \lambda \mid \leq r\}$. Also, let $A_{r}=\{\lambda \in \mathbb{C}|r<| \lambda \mid<1 / r$ for $r \in(0,1)\}$ be an open annulus of containing $S^{1}$. We denote $\overline{A_{r}}$ as the closure of $A_{r}$. Furthermore, let $E_{r}=\{\lambda \in \mathbb{C}|r<| \lambda \mid\}$ be the exterior of the circle $C_{r}$.

For any $r \in(0,1] \subset \mathbb{R}$, we consider the untwisted loop algebra and loop group:

$$
\begin{aligned}
\Lambda_{r} \operatorname{sl}(2, \mathbb{C}) & =\left\{\alpha: C_{r} \rightarrow \operatorname{sl}(2, \mathbb{C}) \mid \alpha \text { is continous }\right\}, \\
\Lambda_{r} S L(2, \mathbb{C}) & =\left\{g: C_{r} \rightarrow S L(2, \mathbb{C}) \mid g \text { is continous }\right\} .
\end{aligned}
$$

We now define special subgroups of $\Lambda S L(2, \mathbb{C})$. The $S U(2) r$-loop group is

$$
\begin{aligned}
\Lambda_{r} S U(2)=\left\{F(\lambda) \in \Lambda_{r} S L(2, \mathbb{C}) \mid\right. & F(\lambda) \in S U(2), \text { for all } \lambda \in S^{1}, \\
& \left.F(\lambda) \text { extends holomorphically to } A_{r}\right\} .
\end{aligned}
$$


Note that the definition of $\Lambda_{r} S U(2)$ implies that $F$ is continuous on $\overline{A_{r}}$ and holomorphic on $A_{r}$. We define the plus loop group as follows:

$$
\begin{array}{r}
\Lambda_{r, B}^{+} S L(2, \mathbb{C})=\left\{W_{+} \in \Lambda_{r} S L(2, \mathbb{C}) \mid W_{+}(\lambda)\right. \text { extends holomorphically to } \\
\left.D_{r} \text { and } B(0) \in \boldsymbol{B}\right\},
\end{array}
$$

where $\boldsymbol{B}$ is a subgroup of $S L(2, \mathbb{C})$. If $\boldsymbol{B}=\{\mathrm{id}\}$ we write the subscript $*$ instead of $\boldsymbol{B}$; if $\boldsymbol{B}=S L(2, \mathbb{C})$ we abbreviate $\Lambda_{r, B}^{+} S L(2, \mathbb{C})$ by $\Lambda_{r}^{+} S L(2, \mathbb{C})$. From now on we will use the subscript $\boldsymbol{B}$ as above only if $\boldsymbol{B} \cap S U(2)=\{$ id $\}$ holds. When $r=1$, we always omit the 1 . In order to make the above groups and algebras complex Banach Lie groups and Lie algebras, we restrict the occurring matrix coefficients to the "Wiener algebra"

$$
\mathcal{A}=\left\{f(\lambda)=\sum_{n \in \mathbb{Z}} f_{n} \lambda^{n}: C_{r} \rightarrow \mathbb{C} ; \sum_{n \in \mathbb{Z}}\left|f_{n}\right|<\infty\right\} .
$$

We will assume from here on out that all matrix coefficients are contained in the Wiener algebra $\mathcal{A}$. It is well known that the Wiener algebra is a Banach algebra relative to the norm $\|f\|=\sum\left|f_{n}\right|$, and that $\mathcal{A}$ consists of continous functions. Moreover, with coefficients in $\mathcal{A}$, the loop groups and loop algebras are Banach Lie groups and Banach Lie algebras. From [2], we quote the following decomposition theorem:

Theorem 2.1 (Iwasawa decomposition, [2]). For any $r \in(0,1]$ and each upper triangular subgroup $\boldsymbol{B}$ of $S L(2, \mathbb{C})$, which satisfies $S U(2) \cdot \boldsymbol{B}=S L(2, \mathbb{C})$ and $S U(2) \cap \boldsymbol{B}=\{\mathrm{id}\}$, the multiplication map

$$
\Lambda_{r} S U(2) \times \Lambda_{r, B}^{+} S L(2, \mathbb{C}) \rightarrow \Lambda_{r} S L(2, \mathbb{C})
$$

is a real analytic diffeomorphism onto.

2.2. Extended framings. It is well known that the Gauss map of an immersion from some Riemann surface into $\mathbb{R}^{3}$ is harmonic if and only if the immersion has constant mean curvature. Let $\mathcal{R}$ be some Riemann surface, and let $S^{2}=S U(2) / U(1)$ be a unit sphere. We consider the following map:

$$
N: \mathcal{R} \rightarrow S^{2}=S U(2) / U(1) .
$$

Then there always exists the lift of $N$ on the universal cover $\tilde{\mathcal{R}}$ of $\mathcal{R}$, which we denote by $F: \tilde{\mathcal{R}} \rightarrow S U(2)$. Then $\alpha=F^{-1} d F$ satisfies the Maurer-Cartan equation:

$$
d \alpha+\frac{1}{2}[\alpha \wedge \alpha]=0 .
$$

Let $\operatorname{sl}(2, \mathbb{C})=\mathfrak{k} \oplus \mathfrak{m}$ be the Cartan decomposition. Then we have

$$
\alpha=\alpha_{0}+\alpha_{1}, \quad \alpha_{0} \in \mathfrak{k}, \quad \alpha_{1} \in \mathfrak{m} .
$$

We also decompose $\alpha_{1}$ as $\alpha_{1}=\alpha_{1}^{\prime}+\alpha_{1}^{\prime \prime}$, where $\alpha_{1}^{\prime}$ and $\alpha_{1}^{\prime \prime}$ are a $(1,0)$-part and a $(0,1)$-part on $\tilde{\mathcal{R}}$, respectively. We introduce the spectral parameter $\lambda \in S^{1}$ by

$$
\alpha_{\lambda}=\lambda^{-1} \alpha_{1}^{\prime}+\alpha_{0}+\lambda \alpha_{1}^{\prime \prime} .
$$

From [2], we quote the following theorem: 
Theorem $2.2([2])$. Let $\tilde{\mathcal{R}}$ be a simply connected Riemann surface. Then a map $N: \tilde{\mathcal{R}} \rightarrow S^{2}$ is harmonic if and only if there exists $\tilde{F}: \tilde{\mathcal{R}} \rightarrow \Lambda_{r} S U(2)$ such that $\tilde{F}^{-1} d \tilde{F}=\lambda^{-1} \alpha_{1}^{\prime}+\alpha_{0}+\lambda \alpha_{1}^{\prime \prime}$ and $\pi \circ \tilde{F}=N$, where $\lambda^{-1} \alpha_{1}^{\prime}+\alpha_{0}+\lambda \alpha_{1}^{\prime \prime}$ is defined in (2.4) and $\pi: S U(2) \rightarrow S^{2}=S U(2) / U(1)$ is the natural projection.

We call $\tilde{F}$ the extended framing of $N$ and denote by $\mathcal{F}_{r}(\tilde{\mathcal{R}})$ the set of extended framings of a simply connected Riemann surface $\tilde{\mathcal{R}}$. From now on, we always use the symbol $F$ for the extended framing of $N$ instead of $\tilde{F}$.

2.3. Sym formula. In this subsection, we show that the CMC surface in any of the three space forms can be recovered from an extended framing, the so-called Sym-Bobenko formula [3]. We denote $\partial_{\lambda}=\partial / \partial \lambda$.

Theorem $2.3([3])$. Let $\tilde{\mathcal{R}}$ be a simply connected Riemann surface and $F_{\lambda} \in \mathcal{F}_{r}(\tilde{\mathcal{R}})$ an extended framing for some $r \in(0,1]$.

(i) Let $H \in \mathbb{R}^{*}$. Then for each $\lambda \in S^{1}$, the map $f: M \times S^{1} \rightarrow \mathbb{R}^{3}$ defined by

$$
f_{\lambda}=-2 i \lambda H^{-1}\left(\partial_{\lambda} F_{\lambda}\right) F_{\lambda}^{-1}
$$

is a conformal CMC-immersion $M \rightarrow \mathbb{R}^{3}$ with $H \neq 0$. by

(ii) Let $\mu \in S^{1}, \mu \neq 1$. Then for each $\lambda \in S^{1}$, the map $f: M \times S^{1} \rightarrow S^{3}$ defined

$$
f_{\lambda}=F_{\mu \lambda} F_{\lambda}^{-1}
$$

is a conformal CMC-immersion into $S^{3}$ with $H=i(1+\mu) /(1-\mu)$.

(iii) For $s \in[r, 1)$ and any $\lambda \in C_{s}$, the map $f: M \times C_{s} \rightarrow H^{3}$ defined by

$$
f_{\lambda}=F_{\lambda}{\overline{F_{\lambda}}}^{t}
$$

is a conformal CMC-immersion of $M$ into $H^{3}$ with $H=\left(1+s^{2}\right) /\left(1-s^{2}\right)$.

2.4. Dressing actions. Let $F \in \Lambda_{r} S U(2)$ be the extended framing of some CMC surface, and let $h_{+}$be an element in $\Lambda_{r}^{+} S L(2, \mathbb{C})$ depending only on $\lambda$. We define

$$
h_{+} \#_{r} F=\tilde{F},
$$

where $h_{+} F=\tilde{F} \tilde{B}$ is the Iwasawa decomposition of $h_{+} F . h_{+} \#_{r} F$ defines an action on the set of the extended framings (see also [9]). We call $h_{+} \#_{r} F$ the dressing action by $h_{+}$.

2.5. Period problems. Let $\mathcal{R}$ be some Riemann surface. We consider the universal cover $\tilde{\mathcal{R}}$ of $\mathcal{R}$ and let $\Delta$ denote the group of deck transformations. For each $\tau \in \Delta$, we define the monodromy matrix $M_{\tau}$ of the extended framing $F$ as $\tau^{*} F=M_{\tau} F k$, where $k \in C^{\infty}(\tilde{\mathcal{R}}, U(1))$. We introduce the following theorem to solve the period problems in $\mathbb{R}^{3}, S^{3}$ or $H^{3}$, respectively, as in [3].

Theorem 2.4 ([3]). Assume $M_{\tau} \in \Lambda_{r} S U(2)$ is the monodromy matrix of some extended framing $F \in \Lambda_{r} S U(2)$ about some $\tau \in \Delta$. Let $f$ be one of the SymBobenko formulas (2.5), (2.6) or (2.7) for $F$, respectively. Then $\tau^{*} f=f$ holds at some $\lambda=\lambda_{0}$ if and only if

(1) $\mathbb{R}^{3}$ case : $\left.M_{\tau}\right|_{\lambda_{0}}= \pm$ id and $\left.\partial_{\lambda} M_{\tau}\right|_{\lambda_{0}}=0$,

(2) $S^{3}$ case: $\left.M_{\tau}\right|_{\lambda_{0}}=\left.M_{\tau}\right|_{\mu \lambda_{0}}= \pm \mathrm{id}$,

(3) $H^{3}$ case : $\left.M_{\tau}\right|_{\lambda_{0}}= \pm$ id. 
2.6. Delaunay surfaces. We now compute the extended framing $F_{D}$ of Delaunay surfaces in space forms in terms of elliptic functions according to [3]. First, let $A \in \Lambda_{r} s l(2, \mathbb{C})$ be the following loop:

$$
A=\left(\begin{array}{cc}
0 & a \lambda^{-1}+b \\
a \lambda+b & 0
\end{array}\right)
$$

where $a, b \in \mathbb{R}^{*},|b| \leq|a|$ and $(a+b)^{2}+\Upsilon=1 / 4$. Here $\Upsilon$ is the following:

$$
\left\{\begin{array}{l}
\Upsilon=0 \quad \text { for the } \mathbb{R}^{3} \text { case, } \\
\Upsilon=-4 a b \sin ^{2}(\theta), \theta \in(0, \pi / 2], \quad \text { for the } S^{3} \text { case, } \\
\Upsilon=-4 a b \sinh ^{2}(q / 2), q \in \mathbb{R}^{*}, \quad \text { for the } H^{3} \text { case. }
\end{array}\right.
$$

Theorem $2.5([3])$. Let $\Phi: \mathbb{C} \rightarrow \Lambda_{r} S L(2, \mathbb{C})$ be defined by $\Phi=\exp ((x+i y) A)$, with $A$ given as in (2.8). Then the extended framing $F_{D}$ of a Delaunay surface in $\mathbb{R}^{3}, S^{3}$ or $H^{3}$ for any $r \in(0,1]$ is given by

$$
F_{D}=\Phi \exp (-\mathbf{f} A) B_{1}^{-1},
$$

where the functions $v=v(x), \mathbf{f}=\mathbf{f}(x)$ and the matrices $B_{0}, B_{1}$ satisfy

$$
\begin{aligned}
v^{\prime 2} & =-\left(v^{2}-4 a^{2}\right)\left(v^{2}-4 b^{2}\right), v(0)=2 b, \\
\mathbf{f} & =\int_{0}^{x} \frac{2 d t}{1+(4 a b \lambda)^{-1} v^{2}(t)}, \\
B_{0} & =\left(\begin{array}{cc}
2 v(b+a \lambda) & -v^{\prime} \\
0 & 4 a b \lambda+v^{2}
\end{array}\right), \quad B_{1}=\left(\operatorname{det} B_{0}\right)^{-1 / 2} B_{0} .
\end{aligned}
$$

Remark 2.6. We have the following explicit form for $v(x)$ using the Jacobi elliptic function:

$$
v(x)=2 b \cdot \operatorname{sn}\left(2 i a\left(x-\frac{i K}{2 a}\right), \frac{b^{2}}{a^{2}}\right)=\frac{2 b}{\operatorname{dn}\left(2 a x, 1-b^{2} / a^{2}\right)},
$$

where sn, $\mathrm{dn}$ are Jacobi elliptic functions and $K=K\left(b^{2} / a^{2}\right)$ is the quarter-period of $\operatorname{sn}\left(x, b^{2} / a^{2}\right)$.

Remark 2.7. We note that the Sym-Bobenko formula (2.5) produces Delaunay surfaces at $\lambda=\lambda_{0}=1$ (resp. $\lambda=\lambda_{0}=e^{i \theta}, \mu=e^{2 i \theta}$ and $\lambda=\lambda_{0}=e^{q}$ ) in $\mathbb{R}^{3}$ (resp. $S^{3}$ and $H^{3}$ ).

Remark 2.8. The choice of zero diagonals of $A$ in (2.8) implies that the Delaunay surface produced by $A$ has a specific axis. In general, the extended framing of a Delaunay surface with any axis is represented by $U F_{D}$, where $U$ is a $z$-independent $\Lambda_{r} S U(2)$ matrix and $F_{D}$ is the Delaunay extended framing produced by $A$ in (2.8).

We now give the definition of the "Delaunay end" of a CMC surface.

Definition 2.1. Let $\overline{\mathcal{R}}$ be a compact Riemann surface and set $\mathcal{R}=\overline{\mathcal{R}} \backslash\left\{p_{1}, \cdots, p_{n}\right\}$, for points $p_{i} \in \bar{R}(i \in\{1, \cdots, n\})$. Let $M$ be $\mathbb{R}^{3}$ (resp. $\left.S^{3}, H^{3}\right)$, let $f: \mathcal{R} \rightarrow M$ be a conformal constant mean curvature immersion $H \neq 0$ (resp. $H \in \mathbb{R}, H>1$ ), and let $f_{D}: \mathcal{S} \rightarrow M$ be some Delaunay surface, where $\mathcal{S}$ is a two-punctured Riemann sphere. If $\lim _{p \rightarrow p_{i}}\left\|f-f_{D}\right\|=0$, then we call the end of $f$ around $p_{i}$ the Delaunay end. 


\section{Asymptotics of CMC SURfaces With Bubbletons}

3.1. CMC surfaces with bubbletons. Let $\pi_{L}: \mathbb{C}^{2} \rightarrow L$ be the hermitian projection onto a line $L \in \mathbb{C P}^{1}$. For $\alpha \in \mathbb{C}$, simple factors ([9]) are given as follows:

$$
\psi_{L, \alpha}=\pi_{L}+\frac{\alpha-\lambda}{1-\bar{\alpha} \lambda} \pi_{L}^{\perp} .
$$

To obtain elements of $\Lambda_{r}^{+} S L(2, \mathbb{C})$, we consider the $Q R$ decomposition for the constant matrix

$$
\left(\operatorname{det} \psi_{L, \alpha}(0)\right)^{-1 / 2} \psi_{L, \alpha}(0)=Q R,
$$

where $Q \in S U(2)$ and $R \in \boldsymbol{B}$. A simple factor of $\Lambda_{r} S L(2, \mathbb{C})$ with $r<|\alpha|$ is a loop of the form

$$
h_{L, \alpha}=\left(\operatorname{det} \psi_{L, \alpha}\right)^{-1 / 2} Q^{-1} \psi_{L, \alpha}
$$

with $\psi_{L, \alpha}$ and $Q$ defined as in (3.1), respectively (3.2). By construction

$$
h_{L, \alpha} \in \Lambda_{s} S U(2)
$$

for $s>|\alpha|$. By Proposition 4.2 in [9] dressing by simple factors is explicit: In fact, for $F(z, \lambda) \in \mathcal{F}_{r}(\tilde{\mathcal{R}}), r \in(0,1)$, and $h_{L, \alpha}$ a simple factor with $\alpha \in \mathbb{C}$ and $r<|\alpha|<1$, we have

$$
h_{L, \alpha} \#_{r} F=h_{L, \alpha} F h_{L^{\prime}, \alpha}^{-1} \text { with } L^{\prime}=\overline{F(z, \alpha)}^{t} L .
$$

The proof can be found in [5].

We now introduce the following definition of CMC surfaces with bubbletons.

Definition 3.1. Let $\mathcal{R}$ be a connected Riemann surface with the universal cover $\tilde{\mathcal{R}}$. Let $F$ be an extended framing defined on $\tilde{\mathcal{R}}$, and let $h_{L, \alpha}$ be a simple factor as in (3.3). We consider $f$ (resp. $\tilde{f}$ ) as the Sym-Bobenko formula defined using $F$ (resp. $h_{L, \alpha} F h_{L^{\prime}, \alpha}^{-1}$ ). Then we call $\tilde{f}$ the CMC surface with a bubbleton of $f$.

3.2. Asymptotics of $\mathrm{CMC}$ surfaces with bubbletons. In this subsection, we show that if an end of a CMC surface is a Delaunay end (see Definition 2.1), then the corresponding end on the CMC surface with bubbletons is again a Delaunay surface. First we note that, for the line $L=\left[a_{0}, b_{0}\right] \in \mathbb{C P}^{1}, \pi_{L}$ and $\pi_{L}^{\perp}$ have the following matrix forms:

$$
\pi_{L}=\frac{1}{\left|a_{0}\right|^{2}+\left|b_{0}\right|^{2}}\left(\begin{array}{cc}
\left|a_{0}\right|^{2} & a_{0} \bar{b}_{0} \\
\bar{a}_{0} b_{0} & \left|b_{0}\right|^{2}
\end{array}\right) \text { and } \pi_{L}^{\perp}=\frac{1}{\left|a_{0}\right|^{2}+\left|b_{0}\right|^{2}}\left(\begin{array}{cc}
\left|b_{0}\right|^{2} & -a_{0} \bar{b}_{0} \\
-\bar{a}_{0} b_{0} & \left|a_{0}\right|^{2}
\end{array}\right) .
$$

We set

$$
\mathfrak{A}=\left\{\alpha \in(-1,1) \mid \frac{b}{a \alpha}>1 \text { and } 1 / 4-\Upsilon+\alpha^{-1} a b(1-\alpha)^{2}>0\right\},
$$

where $a, b$ and $\Upsilon$ are defined in (2.8) and (2.9), respectively. We denote by

$$
\mathcal{U}_{\varepsilon}=\{z=x+i y \in \mathbb{C}: 0<y<\varepsilon\}
$$

a domain in $\mathbb{C}$. We denote a ball with small radius $\varepsilon$ about $\lambda_{0}$ by $B_{\varepsilon}\left(\lambda_{0}\right)$.

We have the following main theorem in this paper: 
Theorem 3.1. Let $\overline{\mathcal{R}}$ be a compact Riemann surface and set $\mathcal{R}=\overline{\mathcal{R}} \backslash\left\{p_{1}, \cdots, p_{n}\right\}$, for points $p_{i} \in \bar{R}(i \in\{1, \cdots, n\})$. Let $M$ be $\mathbb{R}^{3}$ (resp. $\left.S^{3}, H^{3}\right)$, and let $f: \mathcal{R} \rightarrow M$ be a conformal constant mean curvature immersion $H \neq 0$ (resp. $H \in \mathbb{R}, H>1$ ) with Delaunay ends at each $p_{i}$. Let $\tilde{f}$ be its CMC surface with bubbletons defined as in Definition 3.1 with $\alpha \in \mathfrak{A}$, where $\mathfrak{A}$ is defined in (3.7). Then each end of $\tilde{f}_{\lambda_{0}}$ is again a Delaunay end.

Proof. Let $z=x+i y$ be a local coordinate on $\mathcal{R}$ such that $x\left(p_{i}\right)=\infty$ for some $i \in\{1, \cdots, n\}$. Since $f$ has a Delaunay end around $p_{i}$, we have $G \in \mathcal{F}_{r}\left(\mathcal{U}_{\varepsilon}\right)$ such that $\lim _{x \rightarrow \infty} \|$ id $-G^{-1} U F_{D} \|_{B_{\varepsilon}\left(\lambda_{0}\right)}=0$ for some extended unitary Delaunay framing $U F_{D}$ (producing a Delaunay surface at $\lambda_{0}$ ) in any of the three targets, where $F_{D}$ is defined in (2.10) and $U$ is some $z$-independent $\Lambda_{r} S U(2)$ matrix. Let $\tilde{f}$ be the CMC surface with bubbletons obtained from $h_{L, \alpha} \#_{r} G=h_{L, \alpha} G h_{L^{\prime}, \alpha}^{-1}$ with $r<|\alpha|$.

First we show the convergence of the extended framing $h_{L, \alpha} \#_{r} G=h_{L, \alpha} G h_{L^{\prime}, \alpha}^{-1}$ of $\tilde{f}_{\lambda_{0}}$ to some extended framing $h_{L, \alpha} U F_{\mathrm{D}} h_{\infty}^{-1}$ using the following inequality:

$$
\begin{aligned}
\| \mathrm{id}- & \left(h_{L^{\prime}, \alpha} G^{-1} h_{L, \alpha}^{-1}\right)\left(h_{L, \alpha} U F_{\mathrm{D}} h_{\infty}^{-1}\right) \| \\
\leq & \left\|\mathrm{id}-\left(h_{L^{\prime}, \alpha} G^{-1} U F_{\mathrm{D}} h_{L^{\prime}, \alpha}^{-1}\right)\right\| \\
& \quad+\left\|\left(h_{L^{\prime}, \alpha} G^{-1} U F_{\mathrm{D}} h_{L^{\prime}, \alpha}^{-1}\right)\right\|\left\|\mathrm{id}-h_{L^{\prime}, \alpha} h_{L^{\prime \prime}, \alpha}^{-1}\right\| \\
& \quad+\left\|\left(h_{L^{\prime}, \alpha} G^{-1} U F_{\mathrm{D}} h_{L^{\prime \prime}, \alpha}^{-1}\right)\right\|\left\|\mathrm{id}-h_{L^{\prime \prime}, \alpha} h_{\infty}^{-1}\right\|,
\end{aligned}
$$

where $h_{\infty}$ will be represented in (3.16). We recall that $L^{\prime}=\overline{G(z, \alpha)}^{t} L$, and denote $L^{\prime \prime}={\overline{U(\alpha) F_{\mathrm{D}}(z, \alpha)}}^{t} L$. By assumptions, $\lim _{x \rightarrow \infty} \|$ id $-\left(h_{L^{\prime}, \alpha} G^{-1} U F_{\mathrm{D}} h_{L^{\prime}, \alpha}^{-1}\right) \|=0$ and $\lim _{x \rightarrow \infty} \|$ id $-h_{L^{\prime}, \alpha} h_{L^{\prime \prime}, \alpha}^{-1} \|=0$. We show that $h_{L^{\prime \prime}, \alpha}$ converges to $h_{\infty}$. We expand the Delaunay extended framing $F_{\mathrm{D}}$ in Theorem 2.5 to the following matrix form:

$$
F_{\mathrm{D}}=\frac{1}{\mathcal{L}}\left(\begin{array}{cc}
\left(4 a b \lambda+v^{2}\right) C & v^{\prime} C+2 v(b+a \lambda)\left(a \lambda^{-1}+b\right) X^{-1} S \\
\left(4 a b \lambda+v^{2}\right)(a \lambda+b) X^{-1} S & v^{\prime}(a \lambda+b) X^{-1} S+2 v(b+a \lambda) C
\end{array}\right),
$$

where $\mathcal{L}=\sqrt{2 v(b+a \lambda)\left(4 a b \lambda+v^{2}\right)}, \quad X=\sqrt{1 / 4-\Upsilon+\lambda^{-1} a b(1-\lambda)^{2}}, C=$ $\cosh ((x+i y-\mathbf{f}) X), S=\sinh ((x+i y-\mathbf{f}) X)$, and $\mathbf{f}, a, b$ and $\Upsilon$ are defined as in $(2.11),(2.8)$ and $(2.9)$, respectively. We set

$$
\left.F_{\mathrm{D}}\right|_{\lambda=\alpha}=\left(\begin{array}{cc}
\mathcal{A} & \mathcal{B} \\
-\mathcal{B}^{*} & \mathcal{A}^{*}
\end{array}\right) \text {, where } \mathcal{A}^{*}={\overline{\mathcal{A}\left(\bar{\lambda}^{-1}\right)}}^{t} \text { and } \mathcal{B}^{*}={\overline{\mathcal{B}\left(\bar{\lambda}^{-1}\right)}}^{t}
$$

By the conditions in (2.9), the upper-left entry of $F_{\mathrm{D}}$ defined from (3.10) with $\lambda=\alpha$ has no zero, so $\mathcal{A}$ defined in (3.11) has no zero. Thus from (3.6), the entries of $\psi_{L^{\prime \prime}, \alpha}$ as in (3.1) are represented in terms of rational functions of the $\mathcal{B} / \mathcal{A}, \mathcal{B}^{*} / \mathcal{A}$, $\mathcal{A}^{*} / \mathcal{A}$ and their conjugations. Thus the entries of $h_{L^{\prime \prime}, \alpha}$ are represented in terms of $\mathcal{B} / \mathcal{A}, \mathcal{B}^{*} / \mathcal{A}, \mathcal{A}^{*} / \mathcal{A}$ and their conjugations. 
We show that $\mathcal{B} / \mathcal{A}$ (resp. $\mathcal{B}^{*} / \mathcal{A}, \mathcal{A}^{*} / \mathcal{A}$ ) converges to the following periodic function $P$ (resp. $P_{2}, P_{3}$ ) as $x \rightarrow \infty$ :

$$
\begin{gathered}
P=\frac{v^{\prime}+2 v(b+a \alpha)\left(a \alpha^{-1}+b\right) X_{\alpha}^{-1}}{4 a b \alpha+v^{2}}, \\
P_{2}=-(a \alpha+b) X_{\alpha}^{-1} \\
P_{3}=\frac{v^{\prime}(a \alpha+b) X_{\alpha}^{-1}+2 v(b+a \alpha)}{4 a b \alpha+v^{2}},
\end{gathered}
$$

where $X_{\alpha}$ denotes the value $X$ at $\lambda=\alpha$. We show only that $\mathcal{B} / \mathcal{A}$ converges to $P$. (The convergences of $\mathcal{B}^{*} / \mathcal{A}$ and $\mathcal{A}^{*} / \mathcal{A}$ can be argued similarly.) A direct computation shows that $\mathcal{B} / \mathcal{A}$ has the following form:

$$
\begin{aligned}
\frac{\mathcal{B}}{\mathcal{A}} & =\frac{v^{\prime}+2 v(b+a \alpha)\left(a \alpha^{-1}+b\right) X_{\alpha}^{-1} \frac{S_{\alpha}}{C_{\alpha}}}{4 a b \alpha+v^{2}} \\
& =\frac{v^{\prime}+2 v(b+a \alpha)\left(a \alpha^{-1}+b\right) X_{\alpha}^{-1} \frac{1-e^{-2\left(x+i y-\mathbf{f}_{\alpha}\right) X_{\alpha}}}{1+e^{-2\left(x+i y-\mathbf{f}_{\alpha}\right) X_{\alpha}}}}{4 a b \alpha+v^{2}},
\end{aligned}
$$

where $C_{\alpha}$ (resp. $S_{\alpha}, \mathbf{f}_{\alpha}$ ) denotes the value $C$ (resp. $\left.S, \mathbf{f}\right)$ at $\lambda=\alpha$. Thus the following claim proves that $\mathcal{B} / \mathcal{A}$ converges to $P$ :

$$
\lim _{x \rightarrow \infty} \Re\left(\left(x+i y-\mathbf{f}_{\alpha}\right) X_{\alpha}\right)=\infty .
$$

By choosing $\alpha$ in (3.7), $\Re\left(\left(x+i y-\mathbf{f}_{\alpha}\right) X_{\alpha}\right)=\left(x-\mathbf{f}_{\alpha}\right) X_{\alpha}$. We consider the derivative of $\left(x-\mathbf{f}_{\alpha}\right) X_{\alpha}$ with respect to $x$. Because of the property $0<\operatorname{dn}\left(2 b x, 1-a^{2} / b^{2}\right) \leq 1$ for the elliptic function $\mathrm{dn}$, and because of the choice of $\alpha$, the function $\partial_{x}\left(x-\mathbf{f}_{\alpha}\right) X_{\alpha}=1-\frac{2}{1+b /\left(a \alpha \mathrm{dn}^{2}\right)}$ is positive and uniformly bounded away from infinity for any $x \in \mathbb{R}$. Thus $\Re\left(\left(x+i y-\mathbf{f}_{\alpha}\right) X_{\alpha}\right)$ is infinite at $x=\infty$. We set

$$
\begin{aligned}
& \left.h_{\infty} \text { is } h_{L^{\prime \prime}, \alpha}\left(L^{\prime \prime}=\overline{U(\alpha) F_{\mathrm{D}}(z, \alpha)}\right)^{t}\right) \text { with } \mathcal{B} / \mathcal{A}\left(\operatorname{resp} . \mathcal{B}^{*} / \mathcal{A}, \mathcal{A}^{*} / \mathcal{A}\right) \\
& \text { replaced by } P\left(\operatorname{resp.} P_{2}, P_{3}\right) \text { in }(3.12)(\operatorname{resp} .(3.13),(3.14)) .
\end{aligned}
$$

Thus $\lim _{x \rightarrow \infty} \|$ id $-h_{L^{\prime \prime}, \alpha} h_{\infty}^{-1} \|=0$. We note that $h_{\infty}$ is independent of $y . h_{\infty}$ is also in $\Lambda_{s} S U(2)$ for $s>|\alpha|$, and $\partial_{\lambda} h_{L^{\prime \prime}, \alpha}$ converges to $\partial_{\lambda} h_{\infty}$.

Since $\partial_{x}\left(\frac{1-e^{-2\left(x+i y-\mathbf{f}_{\alpha}\right) X_{\alpha}}}{1+e^{-2\left(x+i y-\mathbf{f}_{\alpha}\right) X_{\alpha}}}\right)$ and $\partial_{y}\left(\frac{1-e^{-2\left(x+i y-\mathbf{f}_{\alpha}\right) X_{\alpha}}}{1+e^{-2\left(x+i y-\mathbf{f}_{\alpha}\right) X_{\alpha}}}\right)$ converge to 0 as $x \rightarrow$ $\infty, \partial_{x}(\mathcal{B} / \mathcal{A}), \partial_{x}\left(\mathcal{B}^{*} / \mathcal{A}\right), \partial_{x}\left(\mathcal{A}^{*} / \mathcal{A}\right), \partial_{y}(\mathcal{B} / \mathcal{A}), \partial_{y}\left(\mathcal{B}^{*} / \mathcal{A}\right)$ and $\partial_{y}\left(\mathcal{A}^{*} / \mathcal{A}\right)$ converge to $\partial_{x} P, \partial_{x} P_{2}, \partial_{x} P_{3}, \partial_{y} P, \partial_{y} P_{2}$ and $\partial_{y} P_{3}$ as $x \rightarrow \infty$, respectively. Then we have $\lim _{x \rightarrow \infty}\left\|\alpha-\alpha_{D}\right\|=0$, where $\alpha=\left(h_{L, \alpha} G h_{L^{\prime}, \alpha}^{-1}\right)^{-1} d\left(h_{L, \alpha} G h_{L^{\prime}, \alpha}^{-1}\right)$ and $\alpha_{D}=$ $\left(h_{L, \alpha} U F_{D} h_{\infty}^{-1}\right)^{-1} d\left(h_{L, \alpha} U F_{D} h_{\infty}^{-1}\right)$.

Let $\alpha_{D}=\sum_{j \in \mathbb{Z}} \alpha_{D, j} \lambda^{j}$ be a Fourier expansion of $\alpha_{D}$ along $S^{1}$, where $\alpha_{D, j}$ are $\operatorname{sl}(2, \mathbb{C})$-valued 1 -forms. Since the entries of $\alpha_{D}$ are periodic functions and $\lim _{x \rightarrow \infty}\left\|\alpha-\alpha_{D}\right\|=0, \alpha_{D, j}=0$ for $j \leq-2$ and $j \geq 2$. The periodic property of $\alpha_{D}$ and $\lim _{x \rightarrow \infty}\left\|\alpha-\alpha_{D}\right\|=0$ also imply that $\alpha_{D}$ has the form as in (2.4). Therefore $h_{L, \alpha} U F_{D} h_{\infty}^{-1}$ defines the extended framing of some conformal CMC immersion.

We denote $f_{D}$ as the Sym-Bobenko formula produced by $h_{L, \alpha} U F_{D} h_{\infty}^{-1}$. From the above arguments, we have

$$
\lim _{x \rightarrow \infty}\left\|\tilde{f}-f_{\mathrm{D}}\right\|_{\lambda=\lambda_{0}}=0 .
$$

Finally, we show that $f_{\mathrm{D}}$ is a Delaunay surface. We show only the $\mathbb{R}^{3}$ case. (The $S^{3}$ and $H^{3}$ cases can be argued similarly.) We consider the translation of the 
domain

$$
x+i y \rightarrow x+i(y+\theta), \text { with } \theta \in \mathbb{R} .
$$

Because $h_{\infty}$ depends only on $x$, the following transformation occurs:

$$
\begin{aligned}
U^{-1}\left(f_{\mathrm{D}}+\right. & \left.2 i H^{-1}(h U)_{\lambda}(h U)^{-1}\right) U \\
& \rightarrow\left[\exp (i \theta A) U^{-1}\left(f_{\mathrm{D}}+2 i H^{-1}(h U)_{\lambda}(h U)^{-1}\right) U \exp (-i \theta A)\right. \\
& \left.\quad-i\left(\partial_{\lambda}(\exp (i \theta A))\right) \exp (-i \theta A)\right]_{\lambda=1} .
\end{aligned}
$$

Clearly $U^{-1}\left(f_{\mathrm{D}}+i(h U)_{\lambda}(h U)^{-1}\right) U$ defines a Delaunay surface, and therefore $f_{\mathrm{D}}$ is also a Delaunay surface.

Remark 3.2. The condition $\mathfrak{A}$ in (3.7) is not a restriction for applications. For many cases, if the CMC surface with bubbletons has the same topology as the original CMC surface, then the condition $\mathfrak{A}$ is automatically satisfied.

\section{EXAMPLES}

4.1. Asymptotics of Delaunay surfaces with bubbletons. Let $F_{D}$ be a Delaunay extended framing as in (2.10). From [6], we now choose $\alpha$ as follows:

$$
\alpha=\left(\frac{\delta+2-\sqrt{\delta(\delta+4)}}{2}\right)^{\operatorname{sign}(a b)} \quad \text { with } \delta=\frac{1}{a b}\left(\frac{k^{2}-1}{4}+\Upsilon\right)
$$

where $k^{2} \geq \max \{-16 a b-4 \Upsilon+1,-4 \Upsilon+1,4\}$ and $k \in \mathbb{N}$, and $a, b$ and $\Upsilon$ are defined as in (2.8) and (2.9), respectively. We note that $\operatorname{sign}(a b)$ is +1 (resp. -1 ) if the end is embedded, i.e. $a b>0$ (resp. non-embedded, i.e. $a b<0$ ).

From [6], we have the following existence of the Delaunay surfaces with bubbletons for which the topology is preserved:

Theorem 4.1 ([6]). Let $M$ be $\mathbb{R}^{3}$ (resp. $S^{3}, H^{3}$ ), and let $U F_{D}$ be the Delaunay extended framing of some Delaunay surface $f_{D}: S^{2} \backslash\left\{p_{1}, p_{2}\right\} \rightarrow M$, where $U$ is a $z$-independent $\Lambda_{r} S U(2)$ matrix and $F_{D}$ is defined in (2.10). Let $\tilde{f}_{D}$ be the Delaunay surface with bubbletons defined by $h_{L, \alpha} F_{D} h_{L^{\prime}, \alpha}^{-1}$, where $\alpha$ satisfies (4.1). Then the Delaunay surface with bubbletons $\tilde{f}_{D}$ preserves the topology, i.e. $\tilde{f}_{D}$ is well defined on $S^{2} \backslash\left\{p_{1}, p_{2}\right\}$.

We now set $\alpha$ as in (4.1). Clearly $\alpha \in(-1,1)$ and $1 / 4-\Upsilon+\alpha^{-1} a b(1-\alpha)^{2}$ equals $k^{2} / 4$, and thus the second condition of $\mathfrak{A}$ in (3.7) is satisfied. And we can also show that $b /(a \alpha)>1$, thus the conditions of $\mathfrak{A}$ in (3.7) are satisfied.

Example 4.1. We retain the assumptions in Theorem 4.1. Then both ends of $\tilde{f}_{D}$ again converge to Delaunay surfaces.

In Figure 2, the left (resp. middle, right) image is a cylinder, which has two flat ends, with bubbletons in $\mathbb{R}^{3}$ (resp. $S^{3}, H^{3}$ ). Here, we use the stereographic projection and the Poincare ball model for visualizing surfaces in $S^{3}$ and $H^{3}$, respectively. 

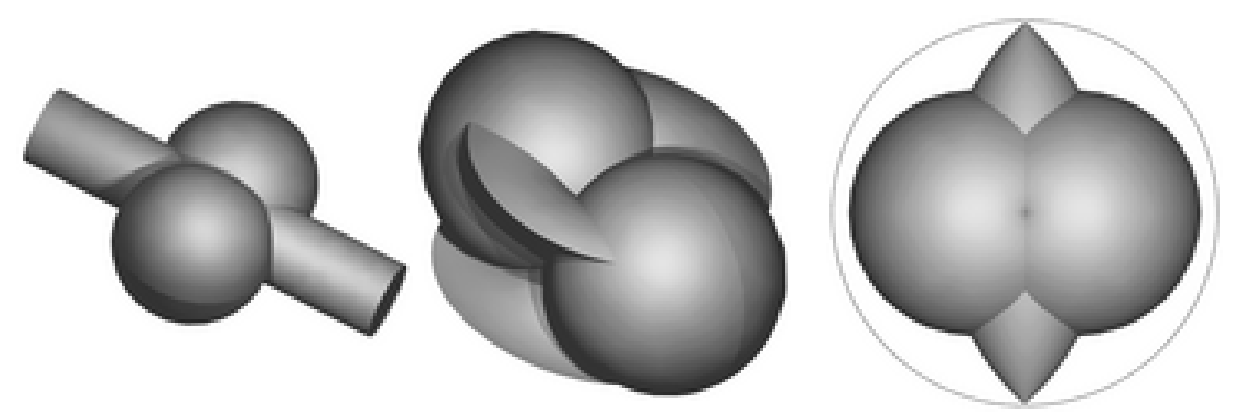

Figure 2. Cylinders with bubbletons in $\mathbb{R}^{3}, S^{3}$ and $H^{3}$. Images are created by Nick Schmitt using CMCLab [7].

4.2. Asymptotics of trinoids with bubbletons. $n$-noids $(n \geq 3)$ are conformal CMC-immersions from $n$-punctured Riemann spheres into any of the three space forms (see [3]). Recently in [4], by using our main result here (Theorem 3.1), it was shown that the CMC $n$-noids $(n=3)$ in the three space forms have Delaunay ends.

In [5], the existences of $n$-noids with bubbletons which preserve the topology of $n$-noids in $\mathbb{R}^{3}$ were shown. In this subsection, we investigate the asymptotics of the ends of such $n$-noids with bubbletons in the case $n=3$ in $\mathbb{R}^{3}$.

We introduce Definition 3.5 in [5]. Let $\mu_{w_{k}}$ be as follows:

$$
\mu_{w_{k}}=\frac{1}{2} \sqrt{1+w_{k}(1-\lambda)^{2} /(4 \lambda)}
$$

where $k \in\{0,1, \infty\}, w_{k} \subset(-\infty, 1] \backslash\{0\}$, and we write $\mu_{k}=\mu_{w_{k}}$. We set

$$
\begin{array}{r}
\Lambda_{w_{0}, w_{1}, w_{\infty}}=\left\{\lambda \in\{0<|\lambda|<1\} \mid 1 / 2 \pm \mu_{0} \pm \mu_{1} \pm \mu_{\infty} \in \mathbb{Z}_{\leq 0},\right. \\
\text { for some choice of signs }\}
\end{array}
$$

We have the following existence theorem for trinoids with bubbletons:

Theorem 4.2 ([5], [4]). Let $f: S^{2} \backslash\{0,1, \infty\} \rightarrow \mathbb{R}^{3}$ be a trinoid with Delaunay ends. Let $\tilde{f}$ be its surface with bubbletons defined by the Sym-Bobenko formula (2.5) using $h_{L, \alpha} F_{D} h_{L^{\prime}, \alpha}^{-1}$, where $\alpha \in \Lambda_{w_{0}, w_{1}, w_{\infty}}$ is defined as in (4.3). Then there exists the trinoid with bubbletons for which the topology is preserved, i.e. $\tilde{f}$ is defined on $S^{2} \backslash\{0,1, \infty\}$.

A direct computation shows that, for some $\alpha$, the conditions (4.1) and (3.7) are compatible. Therefore we obtain the following:

Example 4.2. We retain the assumptions in Theorem 4.2. Further we assume that the $\alpha$ satisfies the condition in (3.7). Then each end of $\tilde{f}$ converges to a Delaunay surface.

In Figure 1, the left (resp. middle, right) image is a triunduloid, which is an immersion from a 3 -punctured sphere and has three embedded Delaunay ends (unduloid type), with bubbletons in $\mathbb{R}^{3}$ (resp. $S^{3}, H^{3}$ ). Here, we again use the stereographic projection and the Poincare ball model for visualizing surfaces in $S^{3}$ and $H^{3}$, respectively. 


\section{ACKNOWLEDGMENTS}

The author thanks Jun-Ichi Inoguchi, Wayne Rossman and Josef Dorfmeister for their suggestions and support.

\section{REFERENCES}

[1] Luigi Bianchi. Vorlesungen über Differentialgeometrie. Leipzig, Berlin, Druck und Verlag von B. G. Teubner, 1910.

[2] J. Dorfmeister, F. Pedit, and H. Wu. Weierstrass type representation of harmonic maps into symmetric spaces. Comm. Anal. Geom., 6(4):633-668, 1998. MR1664887 (2000d:53099)

[3] M. Kilian, S.-P. Kobayashi, W. Rossman, and N. Schmitt. Unitarization of monodromy representations and constant mean curvature trinoids in 3-dimensional space forms $J$. London Math. Soc. (2), 75(3):563-581, 2007.

[4] M. Kilian, W. Rossman, and N. Schmitt. Delaunay asymptotics of constant mean curvature surfaces in space forms via loop group methods. See http://jlms.oxfordjournals.org/cgi/ content/abstract $/ 75 / 3 / 563$

[5] M. Kilian, N. Schmitt, and I. Sterling. Dressing CMC n-noids. Math. Z., 246(3):501-519, 2004. MR2073454 (2005k:53005)

[6] Shimpei Kobayashi. Bubbletions in 3-dimensional space forms. Balkan J. Geom. Appl., 9(2):44-68, 2004. MR2205901 (2007b:53011)

[7] Nicholas Schmitt. cmclab. http://www.gang.umass.edu/software, 2003.

[8] I. Sterling and H. C. Wente. Existence and classification of constant mean curvature multibubbletons of finite and infinite type. Indiana Univ. Math. J., 42(4):1239-1266, 1993. MR1266092 (95a:53015)

[9] Chuu-Lian Terng and Karen Uhlenbeck. Bäcklund transformations and loop group actions. Comm. Pure Appl. Math., 53(1):1-75, 2000. MR1715533 (2000k:37116)

School of Information Environment, Tokyo Denki University, Chiba 270-1382, Japan E-mail address: shimpei@sie.dendai.ac.jp 